\title{
What skilled typists don't know about the QWERTY keyboard
}

\author{
Kristy M. Snyder • Yuki Ashitaka • Hiroyuki Shimada • \\ Jana E. Ulrich • Gordon D. Logan
}

Published online: 8 October 2013

(C) Psychonomic Society, Inc. 2013

\begin{abstract}
We conducted four experiments to investigate skilled typists' explicit knowledge of the locations of keys on the QWERTY keyboard, with three procedures: free recall (Exp.1), cued recall (Exp.2), and recognition (Exp.3). We found that skilled typists' explicit knowledge of key locations is incomplete and inaccurate. The findings are consistent with theories of skilled performance and automaticity that associate implicit knowledge with skilled performance and explicit knowledge with novice performance. In Experiment4, we investigated whether novice typists acquire more complete explicit knowledge of key locations when learning to touchtype. We had skilled QWERTY typists complete a Dvorak touch-typing tutorial. We then tested their explicit knowledge of the Dvorak and QWERTY key locations with the free recall task. We found no difference in explicit knowledge of the two keyboards, suggesting that typists know little about key locations on the keyboard, whether they are exposed to the keyboard for $2 \mathrm{~h}$ or 12 years.
\end{abstract}

Keywords Automaticity - Cognitive control - Automaticity · Implicit/explicit memory

People have poor explicit knowledge of many familiar objects (James, 1890). For example, people are frequently unable to recall the details of coins (Nickerson \& Adams, 1979) or the layout of elevator buttons (Vendetti, Castel, \& Holyoak, 2013)

K. M. Snyder $(\bowtie) \cdot$ J. E. Ulrich • G. D. Logan

Vanderbilt University, Nashville, TN, USA

e-mail: kristy.m.snyder@vanderbilt.edu

G. D. Logan

e-mail: gordon.logan@vanderbilt.edu

Y. Ashitaka $\cdot$ H. Shimada

Kobe University, Hyogo, Japan

K. M. Snyder · G. D. Logan

Department of Psychology, Vanderbilt University,

Nashville, TN 37240, USA and the locations of fire extinguishers (Castel, Vendetti, \& Holyoak, 2013) in the buildings where they work everyday. Their obliviousness to these objects is interesting, but it may not be surprising: People do not need to know which direction the president's head is facing on a coin to spend it, they have ample time to select the appropriate elevator button, and they may never need to use a fire extinguisher. However, some objects, such as the QWERTY keyboard, are used daily and manipulated rapidly. Most college students are skilled typists, who are able to execute five to six keystrokes a second with a high degree of accuracy (Logan \& Crump, 2011). To enable such performance, it seems crucial for typists to know where the keys are located. Yet, previous work has suggested that even complex skills like typewriting are often accomplished without attending to the details of the actions that are being performed (Logan \& Crump, 2009) or the details of the objects that are acted on (Liu, Crump, \& Logan, 2010).

Psychologists suggest that skilled performance does not depend on awareness of the details, because skilled tasks are represented in a qualitatively different manner than novel tasks (Anderson, 1982; Beilock \& Carr, 2001; Fitts \& Posner, 1967; Logan, 1988). Novel tasks require the effortful process of loading explicit knowledge about the task into working memory and manipulating it to support performance. Skilled tasks bypass working memory by relying on automatic procedures that process implicit knowledge. However, this raises questions about the kind of knowledge that underlies expert performance: If experts do not use explicit knowledge to perform skilled tasks, do they lose it? On the one hand, they might. From Ebbinghaus (1964/1895) onward, abundant evidence has indicated that explicit knowledge is forgotten if it is not rehearsed (Underwood, 1957; Wixted, 2004). On the other hand, perhaps they do not lose it, since there is some evidence that memories of well-learned skills are never lost (Bahrick, 1984; Kolers, 1976). Thus, the question is empirical, and the goal of this article was to answer it in the domain of skilled typing.

The present study investigated the explicit knowledge skilled typists have of the locations of keys on the QWERTY 
keyboard. Since the proliferation of personal computers in the 1980s, the QWERTY keyboard has become ubiquitous in western culture. In the United States, middle-school students typically receive formal training on the QWERTY keyboard and type voraciously throughout their teenage years. College students typically have 10 years of typing experience and type 70 words per minute (WPM; Logan \& Crump, 2011). In acquiring this skill, typists must have encoded many memory traces of the QWERTY keyboard from thousands of hours of practice (Ericsson \& Charness, 1994; Logan, 1988). Therefore, it is possible that skilled typists acquired explicit knowledge of the keyboard, which they may retain when they attain high levels of skill. However, hierarchical theories of skilled performance suggest that our knowledge of the details of skilled actions and the objects that we manipulate skillfully is implicit (Liu et al., 2010; Logan \& Crump, 2009; Vallacher \& Wegner, 1987); it is delegated to lower levels of a control hierarchy that are proceduralized and informationally encapsulated (Logan \& Crump, 2011; Shaffer, 1976; Sternberg, Knoll, \& Turock, 1990). Extensive practice may strengthen this implicit knowledge without strengthening explicit knowledge. Novice typists may rely on explicit perception rather than memory. They may look at the keyboard to guide their fingers to the appropriate keys and stop looking at the keyboard when the knowledge becomes implicit (Tapp \& Logan, 2011).

Previous work has demonstrated that skilled typists have poor explicit knowledge of the relative locations of keys on the QWERTY keyboard in tasks that require them to say where one key is located with respect to another (Liu et al., 2010). In the present work, we investigated the extent of skilled typists' explicit knowledge of absolute key locations regardless of the locations of other keys. We assessed their knowledge of absolute key locations in three ways: with a free recall task (Exp.1), a cued recall task (Exp.2), and a recognition memory task (Exp.3). The results indicated that skilled typists have inaccurate and incomplete explicit knowledge of QWERTY key locations. Thus, typists may have forgotten or lost access to the explicit information. Alternatively, they may never have established explicit knowledge of the key locations. We conducted a fourth experiment to determine which explanation is more likely. The Dvorak keyboard (Dvorak, Merrick, Dealey, \& Ford, 1936) is an alternative to the QWERTY keyboard that offers a different arrangement of keys. We introduced skilled QWERTY typists to the Dvorak keyboard by having them complete a 2-h Dvorak touch-typing tutorial. We then assessed the extent of their explicit knowledge of absolute Dvorak key locations with a free recall task.

\section{Experiment 1}

Experiment 1 tested free recall of QWERTY key locations. Typists were given a printout of a blank QWERTY keyboard (see Fig. 1a) and asked to write letters in their correct locations. If they had explicit knowledge of key locations, they should be able to write every letter in its correct location. If they did not have explicit knowledge of key locations, forgot the information, or lost access to it, they should misplace and omit letters.

\section{Method}

Subjects Most modern college students are skilled typists (Logan \& Crump, 2011), so we were able to recruit typists from the Vanderbilt University subject pool, which consists primarily of Vanderbilt students, but also includes some people from the surrounding community. We used the standard Webbased recruitment system and asked for volunteers who had formal training in touch-typing and the self-reported ability to type 40 WPM. The 100 typists that we recruited were 20.8 years old, on average, (range 18-28 years). They had an average of 11.4 years of typing experience and averaged $72.2 \mathrm{WPM}$ on a typing test (Logan \& Zbrodoff, 1998; range 16.4-109.8 WPM; mean accuracy $=93.6 \%$, range $78-100 \%$ ). They received course credit or $\$ 6$ for $30 \mathrm{~min}$ of participation.

Apparatus, stimuli, and procedure Typists first completed a typing history survey. Then they were given a blank QWERTY keyboard printed on a $14 \times 21.6 \mathrm{~cm}$ sheet of paper (Fig. 1a), with the printed side down. When instructed, typists turned the sheet over and filled in the keyboard by writing letters in their appropriate locations. They were given $80 \mathrm{~s}$ to complete the task, divided into four 20 -s time periods. Free recall studies have suggested that $80 \mathrm{~s}$ is enough time to recall 26 items if they were explicitly available (Kahana \& Howard, 2005; Murdock, 1962). To determine when letters were recalled, we had typists write their responses in a different ink color during each 20 -s time period. Typists were not given feedback concerning their accuracy at any time. Once the keyboard task concluded, typists completed a typing test (for details, see Logan \& Zbrodoff, 1998).

\section{Results and discussion}

The probabilities that letters were correctly located, were omitted, or were mislocated are presented on a representation of the keyboard in Fig. 1b. Overall, typists correctly located 14.9 letters $(57.3 \%)$. The remaining letters were either mislocated $(22.8 \%)$ or omitted $(19.6 \%)$. The mean numbers of letters correctly located and mislocated in each of the four time blocks are presented in Fig. 2. The data suggest that typists ran out of explicit knowledge instead of time: From the first to the fourth 20 -s recall period, they correctly located 7.9, $3.5,2.3$, and 1.4 letters. The data also suggest that some of their explicit knowledge was inaccurate, not unavailable: Over the same recall periods, they mislocated $1.3,1.7,1.4$, and 1.3 letters. Accuracy on the free recall task $(M=57.3 \%)$ was significantly 
a

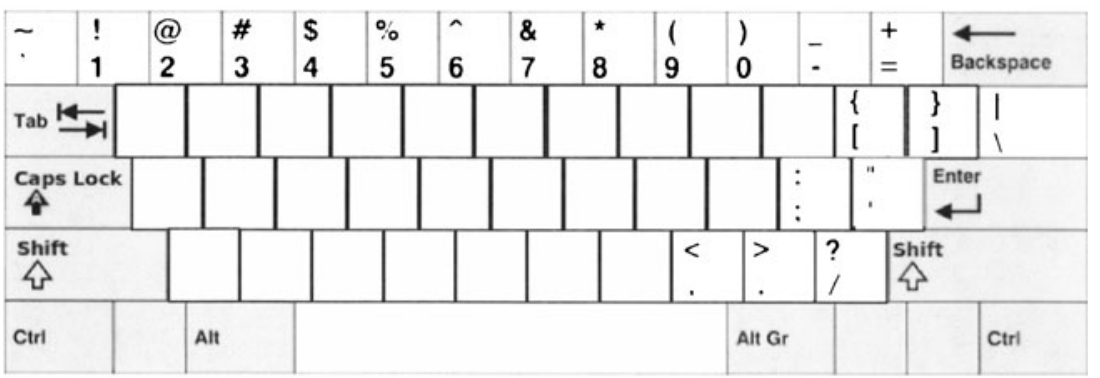

b

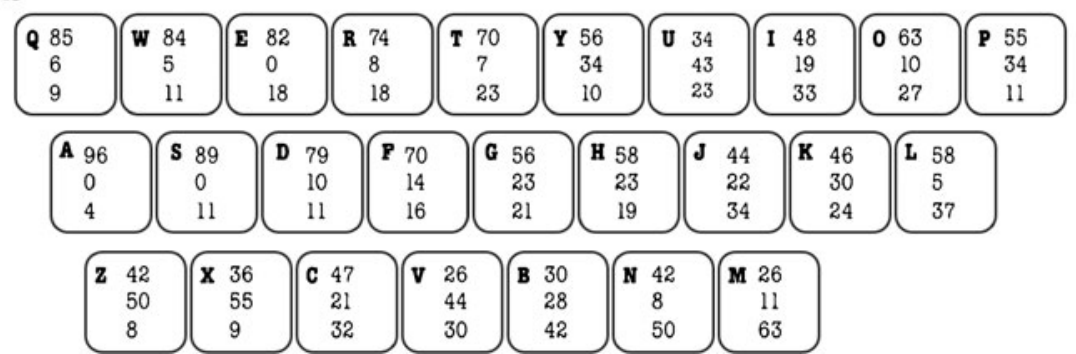

Fig.1 a Schematic of the blank QWERTY keyboard. b Numbers of subjects out of 100 who correctly located (top number), omitted (middle number), and misplaced (bottom number) each letter in Experiment 1

lower than accuracy on the typing test $(M=93.6 \%), t(99)=$ $15.2, p<.001$, which involved a mixture of implicit and explicit knowledge. These results suggest that skilled typists have insufficient explicit knowledge of key locations to support performance on the typing test. Their incomplete explicit knowledge must have been supplemented by excellent implicit knowledge.

Typists' performance on the typing test was not related to their performance on the free recall task. Typing speed was not correlated significantly with correct placements, $r(98)=.16$, $p=.11$, omissions, $r(98)=-.07, p=.49$, or mislocations, $r(98)=-.11, p=.28$. Typing accuracy was not correlated significantly with correct placements, $r(98)=.06, p=.55$, omissions, $r(98)=-.08, p=.43$, or mislocations, $r(98)=-.01$, $p=.94$. These findings suggest that fluent typing does not require explicit knowledge of key locations.

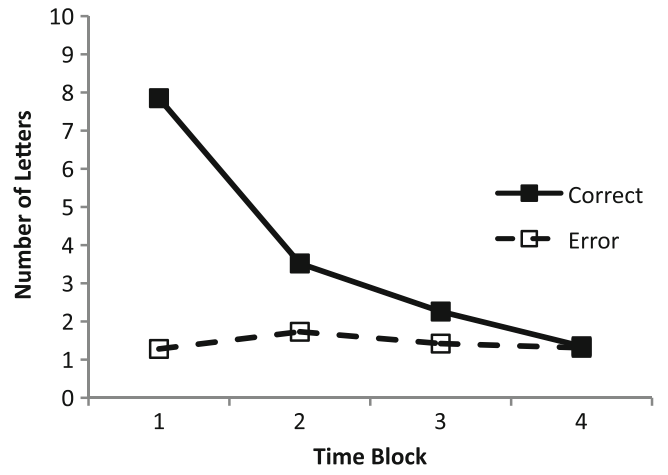

Fig. 2 Numbers of letters correctly placed (solid lines) and misplaced (dashed lines) during each 20-s time period in Experiment $1(1=0-20 \mathrm{~s}$; $2=20-40 \mathrm{~s} ; 3=40-60 \mathrm{~s} ; 4=60-80 \mathrm{~s}$ )

\section{Experiment 2}

Experiment 2 probed skilled typists' explicit knowledge of QWERTY key locations with a cued-recall procedure. We displayed an image of a blank QWERTY keyboard on a computer screen and randomly cued each of the letter keys ten times. Typists were asked to name the letter that corresponded to the cued key. If they have explicit knowledge of key locations, they should be able to identify all of the letters on the keyboard.

Two groups of typists completed the cued recall task. One group completed the task without explicit instruction regarding what they should do with their hands. In debriefing, some of these typists reported that they simulated typing the first time a key's location was cued to help jog their memories. To prevent use of this strategy, we asked a second group of typists to complete the cued recall task while pressing a sequence of keys to suppress motor activity. We called the first group the "nosuppression" group and the second group the "motor suppression" group. Although the no-suppression and motor suppression groups took part in the study sequentially, we considered assignment to groups to be random in our data analyses because the two groups were sampled from the same population.

Method

Subjects Two groups of 16 subjects were recruited from the same population as in Experiment1. For the no-suppression group, the mean age was 23.9 years (range $=20-33$ years), mean typing speed was 73.9 WPM (range 50.1-93.8 WPM), 
and mean accuracy was $93.6 \%$ (range $87.5-98.1 \%$ ). For the motor suppression group, the mean age was 22.2 years (range 18-34 years), mean typing speed was 76.1 WPM (range 37.0115.6 WPM), and mean accuracy was $93.5 \%$ (range 84.9$98.1 \%$ ). All subjects reported having formal touch-typing training. The no-suppression group averaged 13.3 years of typing experience; the motor suppression group averaged 12.3 years of typing experience.

Apparatus, stimuli, and procedure The cued recall procedure was the same for both groups. The experiment was conducted on a MacBook Pro laptop computer with a 15-in. color display that had its keyboard covered. A program, written in METACARD, displayed a $25.4 \times 16.5 \mathrm{~cm}$ gray window in the center of the laptop's screen. A $25.4 \times 10.2 \mathrm{~cm}$ image of a blank QWERTY keyboard (Fig. 1a) was displayed $2.5 \mathrm{~cm}$ from the top of the gray window. Keys were cued by a $1.3 \times$ $1.3 \mathrm{~cm}$ yellow square that outlined the key's location. Each letter key was randomly cued ten times, resulting in 260 trials. Typists were told to say the letter that corresponded to the cued key as quickly and accurately as possible, and to guess if they were unsure. Typists pressed the spacebar of a Korean keyboard connected to the laptop to initiate the next trial. Typists did not receive feedback concerning the accuracy of their performance. Once the experiment concluded, typists completed the typing test (Logan \& Zbrodoff, 1998).

For the no-suppression group, vocal responses were recorded by the laptop's internal microphone. For the motor suppression group, vocal responses were recorded through a microphone that was attached to a set of headphones. A tone was played every $500 \mathrm{~ms}$ through the headphones. Typists were required to press the right arrow key on the Korean keyboard with their right index finger and the left arrow key with their left index finger in a right, right, left, left sequence in time with the tone.

\section{Results and discussion}

No suppression The probabilities of recalling the letter correctly in response to the cue are presented in Fig. 3 as a function of presentation number. Typists correctly identified the cued letter $78.8 \%$ of the time on average. The correlation between overall recall accuracy and typing test accuracy $(M=93.6 \%)$ was not significant, $r(14)=.45, p=.08$. Typing test accuracy was significantly higher than overall recall accuracy, $t(15)=4.7$, $p<.001$, and than recall accuracy on the tenth presentation $(M=82.7 \%), t(15)=3.5, p<.01$. These findings suggest that typists' explicit knowledge was not sufficient to support performance on the typing test. They must have supplemented their explicit knowledge with implicit knowledge. Typing test speed correlated significantly with overall recall accuracy, $r(14)=.51, p=.05$, but not with first-presentation accuracy $(M=67.3 \%), r(14)=.31, p=.24$.

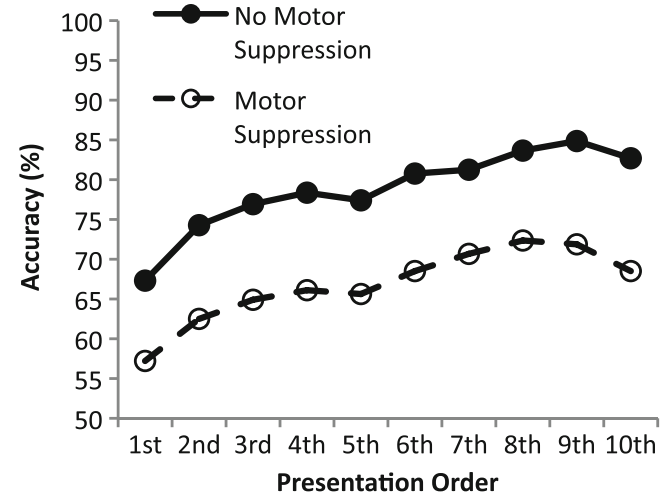

Fig.3 Probabilities of recalling the correct letter on each of the ten times that a key's location was cued, for both the no-suppression (solid line) and motor suppression (dashed line) groups in Experiment 2

Recall accuracy increased significantly from the first to the tenth presentations, $F(9,225)=18.3, M S E=38.5, p<.001$. This suggests that typists either learned the key locations or learned to access their knowledge as they completed the task, despite receiving no feedback. Some typists reported that they simulated typing words the first time that a location was cued in order to refresh their memories of the key locations. Their reaction times (RTs) are consistent with this strategy (see Fig. 4). Correct RTs were longest for the first presentations and decreased over the course of the experiment, $F(9,225)=$ 14.1, $M S E=3,043,195.7, p<.001$.

Motor suppression The probabilities of recalling the cued letter correctly are presented in Fig. 3 as a function of the number of cue presentations. Typists correctly identified the cued letter $66.8 \%$ of the time on average. We observed a significant correlation between overall recall accuracy and typing test accuracy $(M=93.5 \%), r(14)=.50, p=.05$. Typing test accuracy was significantly higher than overall recall accuracy, $t(15)=5.7, p<.001$, and than accuracy on the tenth presentation $(M=68.5 \%), t(15)=4.8, p<.001$.

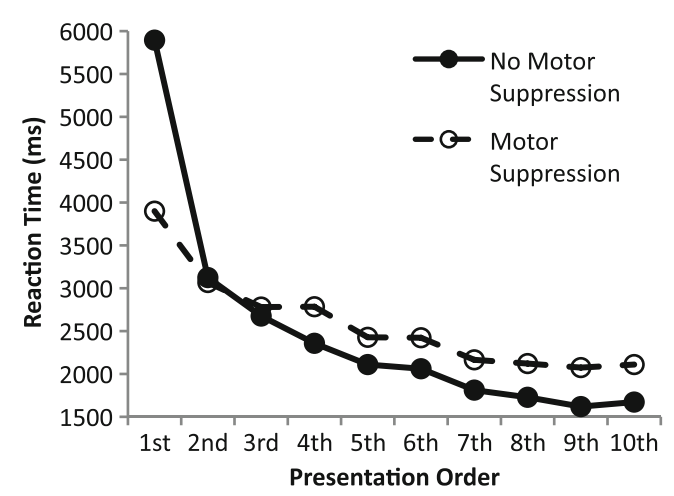

Fig.4 Reaction times for correct responses on each of the ten times that a key's location was cued for the no-suppression (solid line) and motor suppression (dashed line) groups in Experiment 2 
These findings suggest that typists' explicit knowledge was not sufficient to support their performance on the typing test, so they must have supplemented explicit knowledge with implicit knowledge of key location.

Some evidence emerged of a relationship between typing skill and recall accuracy. Typing test speed correlated significantly with overall recall accuracy, $r(14)=.66, p<.01$, and with accuracy on the first presentation $(M=57.2 \%), r(14)=.68$, $p<01$. Recall accuracy improved significantly from the first to the tenth presentations, $F(9,225)=7.3, M S E=76.5, p<.001$. RTs for correct responses are presented as a function of the number of cue presentations in Fig. 4. RTs were longest for the first presentation and decreased over the course of the experiment, $F(9,225)=14.8, M S E=579,123.2, p<.001$.

Effects of motor suppression To assess the effects of motor suppression, we conducted 2 (group: no suppression vs. motor suppression) $\times 10$ (cue presentation) analyses of variance (ANOVAs) on cued recall accuracy and RT. Accuracy was higher in the no-suppression group than in the motor suppression group, $F(1,50)=6.8, M S E=2,709.8, p<.05$, suggesting that having the opportunity to simulate typing helped typists in the no-suppression group to retrieve key locations. This finding is consistent with Logan and Crump's (2011) two-loop theory of skilled typing. Key location information is encapsulated in the inner loop, so the outer loop must monitor the inner loop's output (i.e., hand and finger movements) to discover the location of the keys (Tapp \& Logan, 2011). Overall, RTs did not differ significantly between the groups: Neither the main effect of group, $F(1,50)=0.1, M S E=70,577.5, p=.73$, nor the interaction between group and presentation, $F(9,450)=4.0, M S E=$ $181,159.44, p=.07$, was significant. However, RTs on the first presentation were significantly longer in the nosuppression group ( $M=5,895 \mathrm{~ms})$ than in the suppression group $(M=3,900 \mathrm{~ms}), t(50)=5.3, M S E=7,057,748.2$, $p<.001$. This finding is consistent with reports from typists in the no-suppression group of simulating typing the first time a key was cued to jog their memories.

Recall errors were categorized into adjacent and nonadjacent errors. Adjacent errors were errors in which typists identified a letter whose location was next to the cued key; all other errors were non-adjacent errors. If the typists guessed letters randomly, the expected percentage of adjacent errors would be $16 \%$. However, the observed percentage of adjacent errors was $59.1 \%$. This finding suggests that typists had some knowledge of key locations, although it was not very precise (see also Liu et al., 2010). Typists in the no-suppression group made adjacent errors more often $(M=69.9 \%)$ than did typists in the suppression group $(M=48.3 \%), t(23)=4.6, M S E=$ $3.9, p<.001$. This suggests that typists' knowledge of key locations is more precise when they have the option to simulate typing.

\section{Experiment 3}

The third experiment probed typists' explicit knowledge with a recognition procedure. We displayed an image of a blank QWERTY keyboard and presented a letter in one of the key locations on each trial. Each letter was presented 16 times. Half of the time, the letter was displayed in its correct location (valid condition) and half the time, the letter was displayed in an incorrect location (invalid condition). On half of the invalid presentations (four trials), the letter was displayed adjacent to its correct location (near condition), and on the other half of the invalid presentations (four trials), the letter was displayed in a location that was not adjacent to its correct position ( $\mathrm{far}$ condition). Typists were told to indicate whether or not the letter was presented in its correct location. The contrast between the near and far condition provides some information about the precision of typists' knowledge of letter locations. The more precise their knowledge, the less likely they should be to falsely recognize letter locations in the far condition.

\section{Method}

Subjects A new set of 16 typists was recruited from the same population as before. Their mean age was 24.2 years (range 18-40), mean typing speed was 77.3 WPM (range 37.0-106.7 WPM), and mean accuracy was $95.3 \%$ (range 89.6-100 \%). All typists reported having formal touch-typing training. They had an average of 12.7 years of typing experience.

Apparatus, stimuli, and procedure The computer and displays were the same as Experiment2, except that an uppercase 44-pt Helvetica letter appeared inside one of the key positions on each trial. Each of the 26 letters was displayed in its correct QWERTY keyboard location eight times, in an adjacent key's location four times, and in a nonadjacent key's location four times. In all, 416 trials were presented. The presentation order was randomized for each subject. Typists were asked to indicate whether or not the letter was displayed in its correct location as quickly and as accurately as possible by pressing the left or right arrow key on a Korean keyboard connected to the laptop. A sticker with an uppercase "Y" was placed on the left arrow key, and a sticker with an uppercase "N" was placed on the right arrow key. The letter remained on the screen until typists responded. Then the program initiated the next trial. No feedback about the typists' accuracy was provided. Once the experiment concluded, typists completed the typing test (Logan \& Zbrodoff, 1998).

Results and discussion

Recognition performance was measured by calculating hit rates and false alarm rates. The hit rate was the percentage of valid trials on which typists indicated that the presented letter was 
displayed in its correct location. Figure 5 displays the hit rates for each of the eight times a letter was displayed in its valid location, as well as the false alarm rates for each of the four times a letter was displayed in a near invalid location and each of the four times a letter was displayed in a far invalid location. The overall hit rate $(M=85.0 \%), t(15)=2.5, p<.05$, was significantly lower than the typing test accuracy $(M=95.3 \%)$, but the hit rate on the eighth presentation $(M=86.5 \%)$ was not significantly different from the typing test accuracy, $t(15)=1.6$, $p=.13$. The correlation between the overall hit rate and typing test accuracy was not significant, $r(14)=.10, p=.72$. Typing test speed correlated significantly with both the overall hit rate, $r(14)=.65, p<.01$, and the first-presentation hit rate $(M=82.5 \%), r(14)=.64, p<.01$. These findings suggest that typists with higher levels of skill performed better on the recognition task than did typists with lower levels of skill. Hit rates remained relatively stable from the first to the eighth presentations, $F(7,175)=1.8, M S E=51.2, p=.08$. However, a contrast showed that hit rates were significantly higher on the eighth presentation $(M=86.5)$ than on the first presentation $(M=82.5), F(7,175)=2.5, p<.05$. Additional contrasts showed that the only statistically significant increase in hit rates among successive presentations was between the fourth $(M=82.5)$ and fifth $(M=87.5)$ presentations, $F(7,175)$ $=3.9, p<.001$. RTs for hits decreased significantly from the first to the eighth presentations (see Fig. 6), $F(7,175)=17.9$, $M S E=233,984.5, p<.001$. A contrast showed that hit RTs were significantly shorter on the eighth presentation $(M=$ $1,532.0 \mathrm{~ms})$ than on the first presentation $(M=2,779.7 \mathrm{~ms})$, $F(1,175)=53.2, p<.001$. Additional contrasts revealed that the only statistically significant decrease in hit RTs among

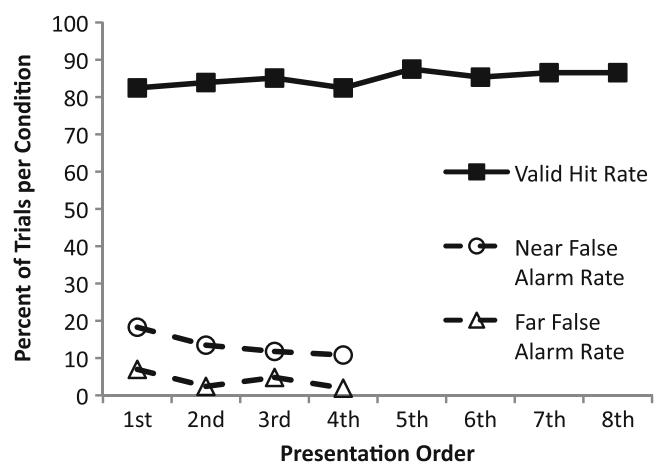

Fig.5 In Experiment 3, each letter was presented 16 times: eight valid presentations (solid line) and eight invalid presentations (dashed lines). Of the eight invalid presentations, four occurred in adjacent, or near, locations, and four occurred in nonadjacent, or far, locations. The 16 presentations of each letter were randomly ordered for each subject, such that the eight valid, four near invalid, and four far invalid trials were equally distributed throughout the experiment. Hit rates (square markers), averaged across letters, are displayed for each of the eight times that a letter was presented in its valid location. False alarm rates, also averaged across letters, are displayed for each of the four times that a letter was presented in a near invalid location (circle markers) and the four times that a letter was presented in a far invalid location (triangle markers)

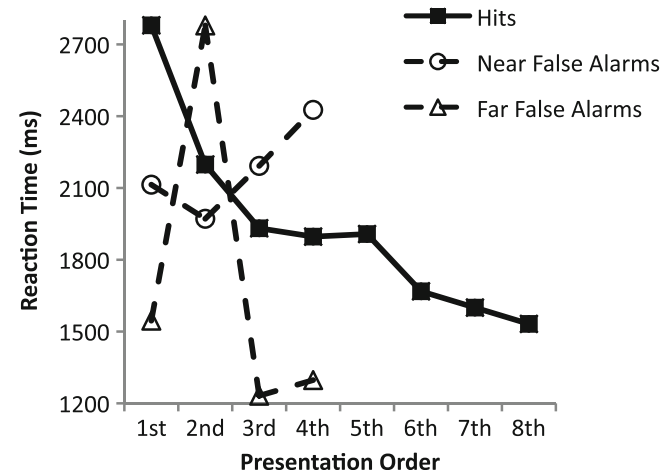

Fig.6 Reaction times, averaged across letters, for hits (square markers), near false alarms (circle markers), and far false alarms (triangle markers) are displayed for each of the eight times a letter was presented in its valid location, each of the four times a letter was presented in a near invalid location, and each of the four times a letter was presented in a far invalid location in Experiment 3

successive presentations was between the first and second $(M=2,197.9 \mathrm{~ms})$ presentations, $F(1,175)=11.6, p<.001$.

The false alarm rates were the percentages of near and far trials on which typists indicated that the presented letter was displayed in its correct location. The overall false alarm rate was $13.6 \%$ for the near condition and $4.0 \%$ for the far condition. A 2 (near vs. far) $\times 4$ (presentations) ANOVA showed that the false alarm rate was higher in the near than in the far condition, $F(1,25)=57.8, M S E=82.2, p<.001$. In the near condition, false alarm rates decreased significantly from the first to the fourth presentations, $F(3,75)=5.1, M S E$ $=55.8, p<.01$. A contrast showed that false alarm rates in the near condition were significantly lower in the fourth presentation $(M=10.8 \%)$ than in the first $(M=18.3 \%), F(1,75)=$ $8.1, p<.01$. Additional contrasts showed no statistically significant decreases in false alarm rates among successive presentations.

In the far condition, false alarm rates also decreased significantly from the first to the fourth presentations, $F(3,75)=$ $6.1, M S E=23.1, p<.001$. A contrast showed that false alarm rates were significantly lower in the fourth presentation (mean difference $1.9 \%)$ than in the first $(M=7.0), F(1,75)=12.5$, $p<.001$. The only statistically significant decrease in far false alarm rates among successive presentations occurred between the first and second (mean difference $=2.4 \%$ ) presentations, $F(1,75)=7.3, p<.01$. These findings suggest that typists have some approximate knowledge of key locations, and that the precision of their knowledge increases with exposure to the task.

\section{Experiment 4}

The previous experiments demonstrated that skilled typists have incomplete and imprecise explicit knowledge of QWERTY key locations. These findings are consistent with 
theories of skill acquisition (Anderson, 1982; Anderson, Fincham, \& Douglass, 1997; Fitts \& Posner, 1967) and automaticity (Logan, 1988). Skill acquisition theories suggest that novice performance relies on algorithms, or task-specific rules. Novices consult the algorithms to complete novel tasks, so the information required for the algorithms must be explicitly accessible in perception or memory. Skilled performance relies on proceduralized knowledge (Anderson, 1982; Anderson et al., 1997; Fitts \& Posner, 1967). Experts retrieve solutions from memory (Logan, 1988), so they have no need to consult the explicit knowledge that supports algorithmic performance. Thus, it is possible that skilled typists forget or lose access to the explicit knowledge they utilized as novices. It is also possible that skilled typists never learned much explicit knowledge in the first place. The explicit knowledge they rely on in the algorithmic stage may be perceptual. Nothing prevents typists from looking at the keyboard when they type, so they may look at the keyboard to find the keys.

The purpose of Experiment 4 was to determine whether novice typists memorize explicit knowledge of key locations when they learn to touch-type. Typing skill is ubiquitous, so finding adults who are novice typists would be difficult (Logan \& Crump, 2011), and children who are just acquiring typing skill would differ from our adult experts in many ways. Instead, we turned skilled QWERTY typists into novices by having them type on the Dvorak keyboard (Dvorak et al., 1936), on which keys occur in unfamiliar locations (see Fig. 7a). We asked typists to complete a Dvorak touch-typing training tutorial that took about $1.5 \mathrm{~h}$ and an additional practice session that lasted about $30 \mathrm{~min}$. We then investigated their explicit knowledge of Dvorak key locations with a free recall task. Typists were given a printout of a blank Dvorak keyboard (see Fig. 7b) and asked to write letters in their correct locations. If typists had established explicit knowledge of the keys' locations, they should be able to fill in the blank keyboard. Afterward, we gave them a free recall task with the QWERTY keyboard to compare their explicit knowledge of the two keyboards.

\section{Method}

Subjects A group of 24 subjects were recruited from the same population as in the previous experiments. All subjects reported having formal QWERTY touch-typing training and averaged 12.7 years of typing experience on the QWERTY keyboard. The mean QWERTY typing speed was 74.5 WPM (range 40.7-107.9 WPM) and accuracy was $93.6 \%$ (range 86.0-99.1\%). Their mean age was 22.4 years (range 18 35 years). All subjects received $\$ 36$ for $3 \mathrm{~h}$ of participation.

Apparatus, stimuli, and procedure The study consisted of two sessions. Session 1 lasted $2 \mathrm{~h}$ and consisted of three tasks: typing history survey, QWERTY typing test, and Dvorak touch-typing tutorial. In the previous experiments, the typing test program had randomly selected one of four possible paragraphs for the typists to transcribe (Logan \& Zbrodoff, 1998). In Experiment4, all typists typed all four paragraphs. The order was counterbalanced using a Latin square. One of the paragraphs was typed on the QWERTY keyboard in Session 1, and the other three paragraphs were typed on the Dvorak keyboard in Session 2. After the paragraph(s) were typed, the typing test program prompted typists to transcribe the sentence "The quick brown fox jumps over the lazy dog," which contains all 26 letters of the alphabet. QWERTY typing speeds did not differ between the paragraph $(M=74.5 \mathrm{WPM})$ and the sentence $(M=$ 76.2 WPM), $t(23)<1, p=.35$. QWERTY typing accuracy also did not differ between the paragraph $(M=93.6 \%)$ and the sentence $(M=89.3 \%), t(23)=1.7, p=.11$.

After the QWERTY typing test, typists completed a Dvorak touch-typing training tutorial that is freely available on the Internet (www.typingweb.com). The interface of the tutorial displayed text that learners were instructed to type at the top of the screen and a schematic of the Dvorak keyboard and figures of hands at the bottom of the screen. The tutorial consisted of 103 exercises. In Exercises 1-21, the home row keys were introduced and practiced. In Exercises 22-31, the top row keys were introduced and practiced. In Exercise 32, the bottom row keys were introduced and practiced. In Exercises 33-103, all keys were practiced. At the beginning of the tutorial, the text prompts displayed letter strings. As more keys were introduced, the text prompts displayed words, and eventually sentences. The average amount of time spent on any given exercise was $93 \mathrm{~s}$ (range 11-280 s). The tutorial progressed to the next exercise when typists achieved at least $70 \%$ accuracy on the current exercise. The typists completed an average of 53.9 exercises (range 37-77) and spent an average of $81.2 \mathrm{~min}$ (range 66-93 $\mathrm{min}$ ) on the Dvorak tutorial. To ensure that all of the keys had been introduced, typists were required to complete at least 34 exercises during Session 1 to be eligible for Session 2.

Session 2 occurred within 2 days of Session 1, lasted $1 \mathrm{~h}$, and consisted of three tasks: Dvorak typing test, Dvorak free recall task, and QWERTY free recall task. Typists first completed the Dvorak typing test. The mean Dvorak typing speed was 15.2 WPM (range 9.5-23.1 WPM) and accuracy was $92.5 \%$ (range $77.2-99.1 \%$ ) when typing the paragraphs. Typing speed did not differ between the paragraphs and the sentence $(M=15.8 \mathrm{WPM}), t(23)=1.8, p=.09$. Typing accuracy also did not differ between the paragraphs and the sentence (both $M \mathrm{~s}=92.4 \%$ ), $t(23)<1, p=.08$.

After the Dvorak typing test, typists completed the Dvorak key location free recall task. Typists were given a blank Dvorak keyboard printed on a $14 \times 21.6 \mathrm{~cm}$ sheet of paper (see Fig. 7b). The rest of the procedures were exactly the same as in Experiment1. After the Dvorak free recall task, typists completed a QWERTY free recall task. The materials and procedures were the same as in Experiment 1 . 
a

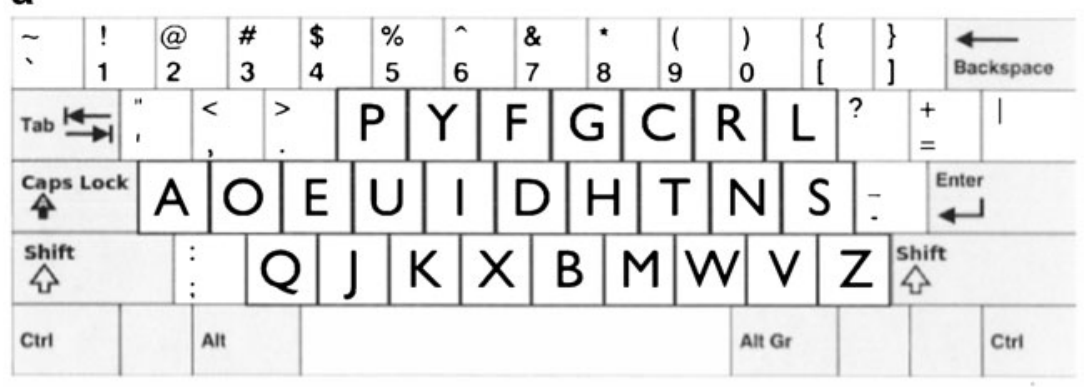

b

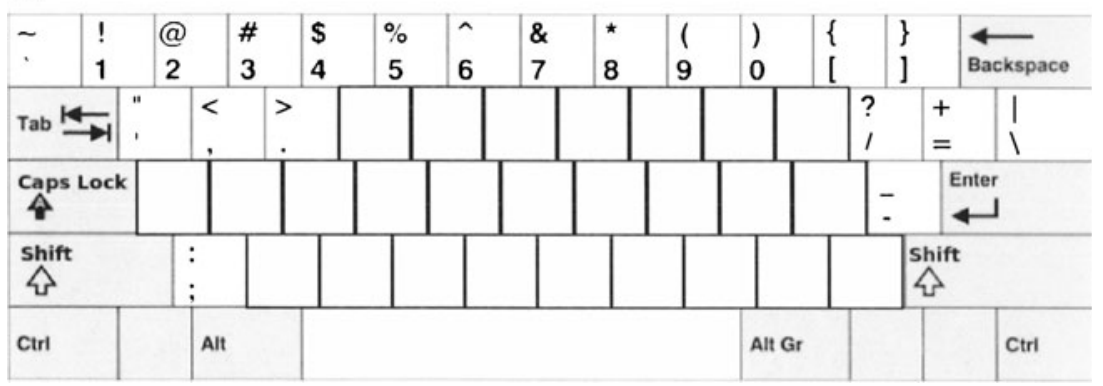

Fig.7 a Schematic of a Dvorak keyboard. b Schematic of the blank Dvorak keyboard used in the Dvorak free recall task in Experiment 4

\section{Results and discussion}

Dvorak analyses Overall, typists correctly located 16.5 letters (63.5\%), mislocated 6.0 letters ( $23 \%$ ), and omitted 4.5 letters $(17.3 \%)$. The mean numbers of letters correctly located and mislocated in the four time blocks are presented in Fig. 8. From the first to the fourth 20 -s recall period, typists correctly located 9.5, 3.7, 2.1, and 1.3 letters. Over the same recall periods, typists mislocated 1.5, 1.2, 1.4, and 1.4 letters. Accuracy on the free recall task $(M=63.6 \%)$ was significantly lower than accuracy on the typing test $(M=92.4 \%), t(23)=5.2, p<.001$.

Typing speed was negatively correlated with correct placements, $r(22)=-.47, p<.05$, positively correlated with mislocations, $r(22)=.41, p<.05$, and unrelated to omissions, $r(22)=.22, p=.31$. These results suggest that typists who completed the typing test quickly performed worse on the free recall task, whereas typists who completed the typing test slowly performed better at recall. Typing test accuracy was negatively correlated with correct placements, $r(22)=-.55$, $p<.01$, was unrelated to mislocations, $r(22)=.26, p=.23$, and was positively correlated with omissions, $r(22)=.41$, $p<.05$. These results indicate that typists' implicit knowledge of key locations did not predict their explicit knowledge of key locations.

QWERTY analyses Typists correctly located 14.1 letters (54.2\%), mislocated 5.9 letters $(22.7 \%)$, and omitted seven letters $(26.9 \%)$. The mean numbers of letters correctly located and mislocated in each of the four time blocks are presented in Fig. 8. From the first to the fourth 20 -s recall periods, typists correctly located $7.3,2.3,2.6$, and 1.9 letters, and mislocated $1.3,2.1,1.3$, and 1.2 letters. Accuracy on the free recall task $(M=54.2 \%)$ was significantly lower than accuracy on the typing test $(M=93.6 \%), t(23)=7.6, p<.001$. Typing speed did not correlate significantly with correct placements, $r(22)=.39, p=.06$, or with mislocations, $r(22)=.06, p=.80$. However, a significant negative correlation was observed between typing speed and omissions, $r(22)=-.41, p<.05$. Typing accuracy did not correlate significantly with correct placements, $r(22)=-.13, p=.55$, mislocations, $r(22)=-.20$, $p=.34$, or omissions, $r(22)=-.03, p=.90$. Overall, the results of the QWERTY free recall task in Experiment4 replicated the results of Experiment1. The only exception was the significant

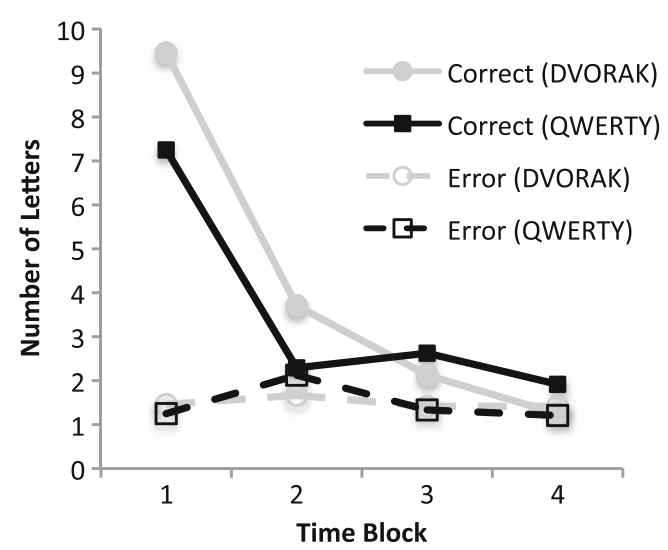

Fig. 8 Numbers of letters correctly placed (solid lines) and misplaced (dashed lines) during each 20-s time period for the Dvorak (gray lines) and QWERTY (black lines) keyboards in Experiment $4(1=0-20 \mathrm{~s} ; 2=20-40 \mathrm{~s}$; $3=40-60 \mathrm{~s} ; 4=60-80 \mathrm{~s})$ 
relationship between faster typing speed and increased letter omissions.

Dvorak versus QWERTY Free recall performance was very similar for the two keyboards. We found no significant difference in the numbers of letters correctly located ( $M=16.5$ for Dvorak, $M=14.1$ for QWERTY), $t(23)=1.9, p=.07$, or the numbers of letters mislocated $(M=6.0$ for Dvorak, $M=5.9$ for QWERTY), $t(23)<1, p=.96$, but the number of omitted letters was lower for Dvorak $(M=4.5)$ than for QWERTY $(M=7.0)$, $t(23)=2.4, p<.05$. These findings suggest that typists have similar explicit knowledge of key locations, whether they spend $2 \mathrm{~h}$ or $12-13$ years interacting with a keyboard. Thus, it appears that typists do not forget or lose access to explicit knowledge of key locations. Instead, they may never have learned it.

\section{General discussion}

In four experiments, we investigated skilled typists' explicit knowledge of the absolute locations of keys on the QWERTY keyboard. Our findings showed that, despite being able to execute six to seven keystrokes per second with near perfect accuracy, skilled typists were able to report the locations of only about half of the keys. They located $57 \%$ (Exp.1) and $54 \%$ (Exp.4) of the letters correctly in free recall, $63 \%$ of the letters correctly in cued recall (Exp.2), and $85 \%$ of the letters correctly in a recognition test (Exp.3). The results are consistent with previous research that has shown that skilled typists have poor explicit knowledge of which hand types each letter (Logan \& Crump, 2009; Snyder \& Logan, 2013; Tapp \& Logan, 2011) or where the keys are relative to each other (Liu et al., 2010). More broadly, the results are consistent with previous research that has shown that people have little explicit knowledge of familiar objects (Castel et al., 2013; James, 1890; Nickerson \& Adams, 1979; Vendetti et al., 2013). Apparently, familiarity and extensive practice does not guarantee awareness of details. This is more surprising for the keyboard than for objects like coins (Nickerson \& Adams, 1979): Typists need to know where the keys are in order to type, but people do not need to know the details of coins in order to spend them. Knowing that a penny is brown is enough.

In each experiment, accuracy on the tests of explicit knowledge was lower than accuracy on the typing test, suggesting that explicit knowledge is not sufficient to support skilled typewriting. The typing test could involve only implicit knowledge, or it could involve a mixture of implicit and explicit knowledge. The explicit knowledge could come from immediate perception of the keyboard or from explicit memory. We suspect that explicit knowledge, if it was used at all, came from perception of the keyboard. Retrieval from explicit memory would be too slow: In Experiment2, cued recall RTs averaged 2,071 and 2,612 ms for the no-suppression and motor suppression groups, respectively. By contrast, Crump and Logan (2010) found that the RT to type a single keystroke in response to a single letter on the screen ranged from 650 to $700 \mathrm{~ms}$ in skilled typists drawn from the same population. Moreover, skilled typists appear to rely on perception of the keyboard to support their performance, in that they type more slowly when their view of the keyboard is blocked (Long, 1976; Rabbitt, 1978; Tapp \& Logan, 2011).

Our results are consistent with theories of automaticity that propose that skilled performance relies on implicit knowledge, whereas novice performance relies on explicit knowledge (Anderson, 1982; Beilock \& Carr, 2001; Beilock, Wierenga, \& Carr, 2002; Fitts \& Posner, 1967; Logan, 1988), and with hierarchical theories of skilled performance that suggest that higher levels use explicit knowledge, whereas lower levels use implicit knowledge (Logan \& Crump, 2011; Shaffer, 1976; Sternberg et al., 1990). Theories of automaticity and hierarchical control do not specify the nature of the explicit knowledge in general; it can vary with the nature of the skill and with the specific models of novice performance. At present, no theories have addressed novice typing. Our results should inform the development of such theories: Experiments 1, 2, 3 suggest that explicit knowledge is forgotten or is inaccessible if it has been learned. Experiment 4 suggests that typists may never have learned it in the first place.

Our results show that daily exposure to an object is not sufficient to produce complete explicit knowledge of the object in memory. Despite the consistency of these findings with previous research and theory, we find it surprising that skilled typists know so little about the keyboard. Typing is a complex task, and directing the fingers to the correct location in the correct order requires an extensive amount of control. Even so, it appears that we are as oblivious to the keyboard as we are to the coins that we spend and the elevator buttons that we push every day.

Author note This research was supported by Grant Nos. BCS 0957074 and BCS 1257272 from the National Science Foundation.

\section{References}

Anderson, J. R. (1982). Acquisition of cognitive skill. Psychological Review, 89, 369-406. doi:10.1037/0033-295X.89.4.369

Anderson, J. R., Fincham, J. M., \& Douglass, S. (1997). The role of examples and rules in the acquisition of a cognitive skill. Journal of Experimental Psychology: Learning, Memory, and Cognition, 23, 932-945. doi:10.1037/0278-7393.23.4.932

Bahrick, H. P. (1984). Semantic memory content in permastore: Fifty years of memory for Spanish learned in school. Journal of Experimental Psychology: General, 113, 1-29. doi:10.1037/00963445.113.1.1

Beilock, S. L., \& Carr, T. H. (2001). On the fragility of skilled performance: What governs choking under pressure? Journal of Experimental Psychology: General, 130, 701-725. doi:10.1037/ 0096-3445.130.4.701 
Beilock, S. L., Wierenga, S. A., \& Carr, T. H. (2002). Expertise, attention, and memory in sensorimotor skill execution: Impact of novel task constraints on dual-task performance and episodic memory. Quarterly Journal of Experimental Psychology, 55A, 1211-1240. doi: $10.1080 / 02724980244000170$

Castel, A. D., Vendetti, M., \& Holyoak, K. J. (2013). Fire drill: Inattentional blindness and amnesia for the location of fire extinguishers. Attention, Perception, \& Psychophysics, 74, 1391-1396. doi:10.3758/s13414-012-0355-3

Crump, M. J. C., \& Logan, G. D. (2010). Hierarchical control over skilled typing: Evidence for word-level control over the execution of individual keystrokes. Journal of Experimental Psychology: Learning, Memory, and Cognition, 36, 1369-1380. doi:10.1037/a0020696

Dvorak, A., Merrick, N. L., Dealey, W. L., \& Ford, G. C. (1936). Typewriting behavior. New York: American Book Co.

Ebbinghaus, H. (1964). Memory: A contribution to experimental psychology (H.A. Ruger \& C.E. Bussenius, Trans.). New York: Dover. Original work published 1895.

Ericsson, K. A., \& Charness, N. (1994). Expert performance: Its structure and acquisition. American Psychologist, 49, 725-747. doi:10.1037| 0003-066X.49.8.725

Fitts, P. M., \& Posner, M. I. (1967). Human performance. Monterey: Brooks/Cole

James, W. (1890). The principles of psychology. New York: Henry Holt.

Kahana, M. J., \& Howard, M. W. (2005). Spacing and lag effects in free recall of pure lists. Psychonomic Bulletin \& Review, 12, 159-164. doi:10.3758/BF03196362

Kolers, P. A. (1976). Reading a year later. Journal of Experimental Psychology: Human Learning and Memory, 2, 554-565. doi:10. 1037/0278-7393.2.5.554

Liu, X., Crump, M. J. C., \& Logan, G. D. (2010). Do you know where your fingers have been? Explicit knowledge of the spatial layout of the keyboard in skilled typists. Memory \& Cognition, 38, 474-484. doi:10.3758/MC.38.4.474

Logan, G. D. (1988). Toward an instance theory of automatization. Psychological Review, 95, 492-527. doi:10.1037/0033-295X.95.4. 492

Logan, G. D., \& Crump, M. J. C. (2009). The left hand doesn't know what the right hand is doing: The disruptive effects of attention to the hands in skilled typewriting. Psychological Science, 20, 12961300. doi:10.1111/j.1467-9280.2009.02442.x
Logan, G. D., \& Crump, M. J. C. (2011). Hierarchical control of cognitive processes: The case for skilled typewriting. In B. H. Ross (Ed.), The psychology of learning and motivation: Advances in research and theory (Vol. 54, pp. 1-27). Burlington: Academic Press.

Logan, G. D., \& Zbrodoff, N. J. (1998). Stroop type interference: Congruity effects in color naming with typewritten responses. Journal of Experimental Psychology: Human Perception and Performance, 24, 978-992. doi:10.1037/0096-1523.24.3.978

Long, J. (1976). Visual feedback and skilled keying: Differential effects of masking the printed copy and the keyboard. Ergonomics, 19, 93-110.

Murdock, B. B., Jr. (1962). The serial position effect of free recall. Journal of Experimental Psychology, 64, 482-488. doi:10.1037/h0045106

Nickerson, R. S., \& Adams, M. J. (1979). Long-term memory for a common object. Cognitive Psychology, 11, 287-307. doi:10.1016/ 0010-0285(79)90013-6

Rabbitt, P. (1978). Detection of errors by skilled typists. Ergonomics, 21, 945-958. doi:10.1080/00140137808931800

Shaffer, L. H. (1976). Intention and performance. Psychological Review, 83, 375-393. doi:10.1037/0033-295X.83.5.375

Snyder, K.M., \& Logan, G.D. (2013). Monitoring-induced disruption in skilled typewriting. Journal of Experimental Psychology: Human Perception and Performance. doi:10.1037/a0031007

Sternberg, S., Knoll, R. L., \& Turock, D. L. (1990). Hierarchical control in the execution of action sequences: Tests of two invariance principles. In M. Jeannerod (Ed.), Attention and performance XIII: Motor representation and control (pp. 3-55). Hillsdale: Erlbaum.

Tapp, K. M., \& Logan, G. D. (2011). Attention to the hands disrupts skilled typewriting: The role of vision in producing the disruption. Attention, Perception, \& Psychophysics, 73, 2379-2383. doi:10. 3758/s13414-011-0208-5

Underwood, B. J. (1957). Interference and forgetting. Psychological Review, 64, 49-60. doi:10.1037/h0044616

Vallacher, R. R., \& Wegner, D. M. (1987). What do people think they're doing? Action identification and human behavior. Psychological Review, 94, 3-15. doi:10.1037/0033-295X.94.1.3

Vendetti, M., Castel, A. D., \& Holyoak, K. J. (2013). The floor effect: Impoverished spatial memory for elevator buttons. Attention, Perception, \& Psychophysics, 75, 636-643. doi:10.3758/s13414-013-0448-7

Wixted, J. T. (2004). The psychology and neuroscience of forgetting. Annual Review of Psychology, 55, 235-269. doi:10.1146/annurev. psych.55.090902.141555 
Reproduced with permission of the copyright owner. Further reproduction prohibited without permission. 